\title{
Short-Term Effect of Percutaneous Bipolar Continuous Radiofrequency on Sacral Nerves in Patients Treated for Neurogenic Detrusor Overactivity After Spinal Cord Injury: A Randomized Controlled Feasibility Study
}

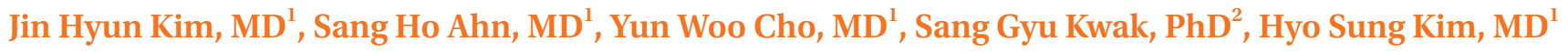

${ }^{1}$ Department of Physical Medicine and Rehabilitation, Yeungnam University College of Medicine, Daegu; ${ }^{2}$ Department of Medical Statistics, Catholic University of Daegu School of Medicine, Daegu, Korea

Objective To investigate the short-term effects of bipolar radiofrequency applied to sacral nerves to treat neurogenic detrusor overactivity in patients with spinal cord injury.

Methods Ten patients with spinal cord injury with neurogenic detrusor overactivity were recruited. These subjects were randomized to two groups: intervention $(n=5)$ and control $(n=5)$, members of which received conventional treatment. Voiding diary, International Consultation on Incontinence Questionnaire (ICIQ) and the urinary incontinence quality of life scale (IQOL) data were obtained and an urodynamic study (UDS) was performed before and after intervention. In the intervention group, percutaneous bipolar continuous radiofrequency (CRF) was performed on both the S2 and S3 nerves in each patient.

Results In a comparison of daily frequency and number of urinary incontinence and ICIQ and IQOL scores at baseline and at 1 and 3 months after intervention, all variables achieved a significant effect for time $(\mathrm{p}<0.05)$. Regarding UDS parameters, pre/post intervention differences between baseline and 3-month post-intervention for volume at maximal detrusor pressure during filling and reflex detrusor volume at first contraction were significantly different between the two groups $(\mathrm{p}<0.05)$. However, pre/post intervention differences in maximum cystometric capacity and maximum detrusor pressure during filling were not significant between the two groups ( $\mathrm{p}>0.05$ ).

Conclusion Percutaneous bipolar CRF applied to sacral nerves might be an effective therapy for neurogenic overactive bladder that reduces urinary incontinence and improves quality of life.

Keywords Radiofrequency, Sacral nerves, Detrusor overactivity, Urinary incontinence, Maximal cystometric capacity 


\section{INTRODUCTION}

In patients with spinal cord injury, impaired urinary bladder function is a major complication. Neurogenic bladder, especially the overactive type, causes bladder dysfunction such as urinary incontinence and reduced bladder capacity, and it lowers quality of life [1]. Additionally, prolonged neurogenic bladder may cause ureterovesical reflux resulting in renal damage, which reduces lifespan [2]. Patients with this symptom should take anticholinergic drugs to suppress detrusor muscle pressure and perform 5 times a day or so to avoid urinary incontinence. However, anticholinergics have side effects such as thirst, memory impairment, reduced sweating, emotional disturbance, constipation, pupil dilatation, visual disturbances, and tachycardia [3], and clean intermittent catherization might cause urethral discomfort and possible urinary tract infections [4]. For these reasons, alternative treatments have been introduced.

Previous studies have reported that selective sacral rhizotomy in patients with neurogenic bladder increased bladder capacity and improved urinary incontinence. The rationale for this procedure was to denervate sacral nerves that were innervating the detrusor muscle to attenuate the bladder spasm and hypertonicity in neurogenic bladder [5-7]. However, this procedure is destructive and is difficult to perform repeatedly. Percutaneous radiofrequency (RF) sacral rhizotomy was first introduced for treating hyperreflexic bladder by Mulcahy and Young $[8,9]$. They reported augmentation of bladder capacity and observed successful preservation of its effect over nine years later. Ferreira reported on the effects of sacral rhizotomy by percutaneous continuous radiofrequency (CRF), which uses high-frequency waves to ablate nerves in patients with hyperactive neurogenic bladder that results in increased maximal cystometric capacity and reduced detrusor pressure at maximal cystometric capacity [10]. Cho and Lee [11] reported that bilateral S2 or S3 monopolar CRF sacral rhizotomy improved bladder capacity and urinary incontinence in patients with spinal cord injury with intolerable neurogenic bladder. They reported that there were no complications such as pain, focal discomfort around the treated area, or sexual dysfunction. However, no reports have been issued regarding the effect of bipolar CRF on sacral nerves in patients with an overactive neurogenic bladder. Therefore, in the present study, which was conducted using a small number of subjects and a randomized controlled design, we investigated the short-term effect of bipolar RF ablation of sacral nerves because of neurogenic detrusor overactivity in spinal cord injury patients.

\section{MATERIALS AND METHODS}

\section{Subjects}

We recruited patients with spinal cord injury (American Spinal Injury Association [ASIA] scale A \& B) with neurogenic detrusor overactivity who had been treated for more than one year. Ten patients were recruited (male 7 , female 3; mean age, $41.2 \pm 8.01$ years). The exclusion criteria were as follows: 1 ) age $<20$ years or $>70$ years; 2 ) previous surgery for bladder or prostate; 3 ) presence of indwelling catheterization; 4) medical problems such as refractory hypertension or cardiovascular diseases; 5) coagulopathies; 6) pregnancy; and 7) ASIA scale C, D, or E. The Institutional Review Board of our hospital approved the study protocol. All involved subjects provided written, informed consent. Patients were allocated to two groups in a $1: 1$ ratio-the intervention group $(n=5)$, members of which received percutaneous bipolar continuous $\mathrm{RF}$ on the S2 and S3 spinal nerves (male 3, female 2; mean age, $42 \pm 8.46$ years; mean disease duration, $91 \pm 31$ months), and the control group $(n=5)$, members of which received conventional medical treatment (male 4, female 1; mean age, $40.4 \pm 8.4$ years; mean disease duration of disease, $56 \pm 32$ months).

\section{Clinical evaluations}

A three-day voiding diary was collected from patients in the intervention group before the procedure and at 2, 4 , 8 and 12 weeks after intervention. The voiding diary consisted of frequency and volume of voiding and frequency and volume of urinary incontinence during one day. In addition, the International Consultation on Incontinence Questionnaire (ICIQ) and the urinary incontinence quality of life scale (IQOL) were administered before intervention and at 2, 4, 8, and 12 weeks after intervention. The ICIQ is a questionnaire that evaluates the symptoms of incontinence, and its reliability and validity have been well-established [12]. The IQOL is a questionnaire that evaluates urinary incontinence and quality of life and is known to have excellent repeatability and validity for 
evaluating quality of life in patients with urinary incontinence $[13,14]$. Patients in the control group were evaluated in the same manner regarding the three-day voiding diary, ICIQ and IQOL.

An urodynamic study (UDS) was performed in the intervention group before and 12 weeks after intervention using Duet LOGIC G2 (Mediwatch Plc, West Palm Beach, FL, USA). UDS provides a useful means of assessing the physiological function and pathology of the lower urinary tract in patients with neurogenic bladder [15]. It measures maximum cystometric capacity, maximum detrusor pressure during filling, volume at maximal detrusor pressure during filling, and reflex detrusor volume at first contraction. For the UDS, the bladder was filled with 24 $\mathrm{mL} / \mathrm{min}$ of normal saline, and detrusor pressure was defined as the difference between intravesical and abdominal pressure. Patients in the control group underwent the urodynamic study at baseline and three months after baseline.

\section{The bipolar continuous radiofrequency procedure}

In a prone position, insertion sites were determined using two curved-tip 10-cm 18-gauge cannulae under fluoroscopic guidance. After these two cannulae were inserted, the tips were placed as close as possible to the S2 or S3 nerves under AP fluoroscopic guidance, and optimal electrode position was confirmed by lateral fluoroscopic imaging to be not beyond the ventral surface of the optimal electrode position or beyond the sacral ventral surface. Sensory nerve stimulation was then performed using an RF generator G4 (Cosman Medical Inc., Burlington, MA, USA). During sensory nerve stimulation, paresthesia, pain and muscle contraction around the sacrum should be observed at $<0.7 \mathrm{~V}$. After the cannulae had been placed in the required position, contrast was injected to confirm the absence of contrast material in the subarachnoid and intravascular spaces. RF was applied for 90 seconds at a tip temperature of $<80^{\circ} \mathrm{C}$. The procedure was performed in this manner at both the S2 and S3 nerve segments (Fig. 1).

\section{Statistical analysis}

SPSS ver. 19.0 software (SPSS Inc., Chicago, IL, USA) was used for the data analysis. To analyze daily mean frequency and volume of urinary incontinence and ICIQ and IQOL scores, we used repeated-measure two-factor analysis, and to analyze differences between baseline and follow-up UDS parameters (maximum cystometric capacity, maximum detrusor pressure during filling, volume at maximal detrusor pressure during filling, and reflex detrusor volume at first contraction), we calculated pre/post intervention differences. The Mann-Whitney U test was used to compare the patient and control groups with respect to the pre/post values of the UDS parameters. Statistical significance was accepted for $\mathrm{p}$-values $<0.05$.

\section{RESULTS}

Table 1 shows the patients' demographic and clinical data including baseline values for UDS parameters, ICIQ and IQOL scores, and daily mean frequency and volume of urinary incontinence.

The comparisons by time, group, and timexgroup re-
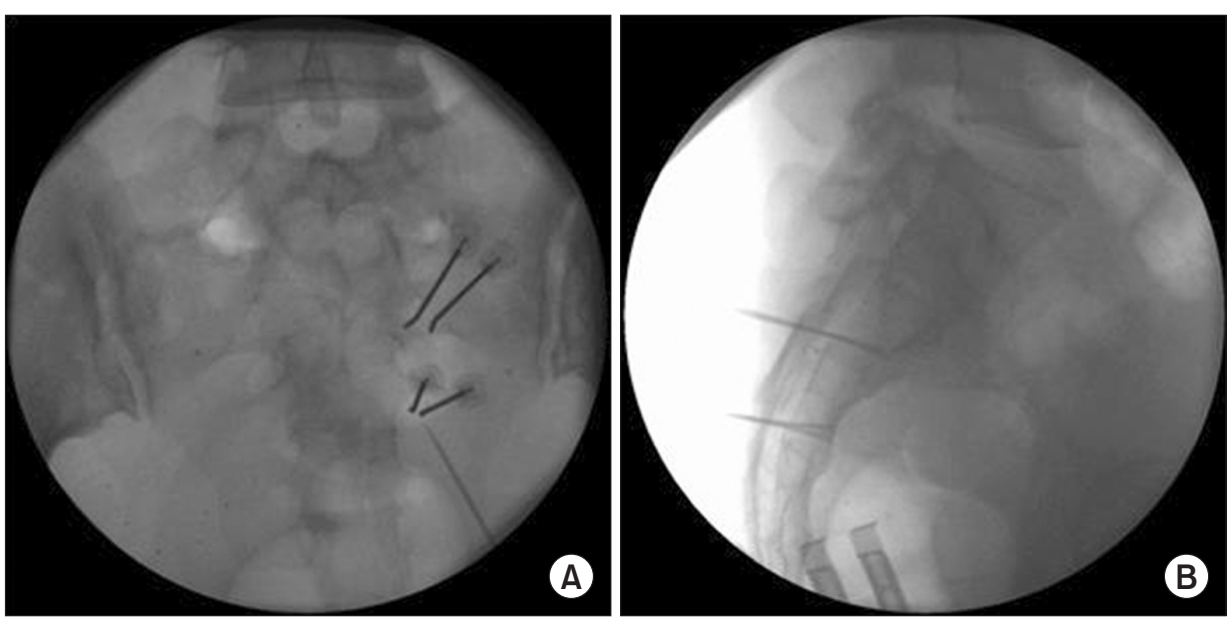

Fig. 1. (A) The anteroposterior view of bipolar radiofrequency on the right S2 and S3 nerve roots. (B) The lateral view of bipolar radiofrequency on the right S2 and S3 nerve roots. 
Table 1. Patients' demographic and clinical data

\begin{tabular}{|c|c|c|c|c|c|c|c|c|c|c|}
\hline \multirow[b]{2}{*}{ Patient no. } & \multirow[b]{2}{*}{ Age/sex } & \multirow[b]{2}{*}{ NLI (AIS) } & \multicolumn{4}{|c|}{ UDS parameter } & \multirow[b]{2}{*}{ ICIQ } & \multirow[b]{2}{*}{ IQOL } & \multicolumn{2}{|c|}{ Incontinence } \\
\hline & & & RDV & MDP & V at MDP & MCC & & & Frequency & $\begin{array}{c}\text { Volume } \\
(\mathrm{mL})\end{array}$ \\
\hline \multicolumn{11}{|l|}{ Intervention } \\
\hline 1 & $\mathrm{M} / 53$ & C4 (A) & 286 & 66 & 344 & 402 & 7 & 101 & 5 & 100 \\
\hline 2 & $\mathrm{M} / 32$ & C5 (B) & 179 & 67 & 595 & 600 & 10 & 85 & 2 & 70 \\
\hline 3 & $\mathrm{~F} / 48$ & C4 (B) & 66 & 77 & 260 & 300 & 12 & 33 & 10 & 57 \\
\hline 4 & $\mathrm{~F} / 40$ & T6 (A) & 209 & 112 & 230 & 500 & 11 & 44 & 15 & 100 \\
\hline 5 & $\mathrm{M} / 37$ & $\mathrm{~T} 12(\mathrm{~A})$ & 253 & 78 & 180 & 360 & 18 & 28 & 6 & 95 \\
\hline \multicolumn{11}{|l|}{ Control } \\
\hline 1 & $\mathrm{M} / 46$ & C4 (A) & 162 & 153 & 189 & 315 & 7 & 100 & 7 & 221 \\
\hline 2 & $\mathrm{M} / 36$ & C6 (A) & 215 & 48 & 298 & 400 & 10 & 37 & 2 & 125 \\
\hline 3 & $\mathrm{~F} / 31$ & T8 (A) & 232 & 41 & 277 & 320 & 12 & 71 & 5 & 150 \\
\hline 4 & $\mathrm{M} / 52$ & T6 (B) & 49 & 50 & 101 & 275 & 12 & 91 & 2 & 120 \\
\hline 5 & $\mathrm{M} / 37$ & $\mathrm{~T} 11(\mathrm{~A})$ & 250 & 31 & 171 & 345 & 17 & 30 & 6 & 90 \\
\hline
\end{tabular}

NLI, neurological level of injury; AIS, American Spinal Injury Association impairment scale; UDS, urodynamic study; MCC, maximum cystometric capacity; MDP, maximum detrusor pressure during filling; RDV, reflex detrusor volume at first contraction; $\mathrm{V}$, volume.

Table 2. Daily mean frequency and volume of urinary incontinence and ICIQ and IQOL scores at baseline and 1 and 3 months after intervention in the two study groups

\begin{tabular}{|c|c|c|c|c|c|c|}
\hline & \multirow{2}{*}{ Baseline } & \multirow{2}{*}{1 mo } & \multirow{2}{*}{3 mo } & \multicolumn{3}{|c|}{ F (p-value) } \\
\hline & & & & Time & Group & Time $\times$ Group \\
\hline \multicolumn{7}{|c|}{$\begin{array}{l}\text { Frequency of urinary } \\
\text { incontinence }\end{array}$} \\
\hline Control & $4.4 \pm 2.30$ & $4.6 \pm 2.79$ & $4.4 \pm 1.82$ & $8.050(0.004)^{*}$ & $0.250(0.63)$ & $8.390(0.003)^{*}$ \\
\hline Intervention & $7.6 \pm 5.03$ & $2.4 \pm 2.88$ & $1.0 \pm 1.73$ & & & \\
\hline \multicolumn{7}{|c|}{$\begin{array}{l}\text { Volume of urinary } \\
\text { incontinence }\end{array}$} \\
\hline Control & $143.2 \pm 47.66$ & $130.4 \pm 74.35$ & $139 \pm 97.32$ & $3.840(0.043)^{*}$ & $10.620(0.012)^{*}$ & $2.670(0.100)$ \\
\hline Intervention & $84.4 \pm 19.73$ & $24.0 \pm 40.72$ & $5.4 \pm 7.80$ & & & \\
\hline \multicolumn{7}{|l|}{ ICIQ } \\
\hline Control & $12.6 \pm 7.77$ & $11.6 \pm 5.41$ & $12.2 \pm 6.57$ & $7.650(0.005)^{*}$ & $0.640(0.446)$ & $5.070(0.020)^{*}$ \\
\hline Intervention & $13.2 \pm 3.27$ & $8.6 \pm 2.79$ & $7.2 \pm 2.59$ & & & \\
\hline \multicolumn{7}{|l|}{ IQOL } \\
\hline Control & $58.2 \pm 32.78$ & $56.0 \pm 19.99$ & $56.8 \pm 24.53$ & $4.722(0.024)^{*}$ & $0.627(0.451)$ & $6.870(0.007)^{*}$ \\
\hline Intervention & $57.8 \pm 26.17$ & $73.8 \pm 25.76$ & $77.0 \pm 22.71$ & & & \\
\hline
\end{tabular}

Values are presented as mean \pm standard deviation.

ICIQ, International Consultation on Incontinence Questionnaire; IQOL, urinary incontinence quality of life scale.

*Statistically significant at the $\mathrm{p}<0.05$.

garding daily mean frequency and volume of urinary incontinence and ICIQ and IQOL scores at baseline and at 1 and 3 months after intervention are shown in Table 2. These variables achieved a significant effect for time $(\mathrm{p}<0.05)$, and for the group $\times$ time interaction, frequency of urinary incontinence and ICIQ and IQOL scores achieved statistical significance. Only daily mean volume of urinary incontinence showed a significant group effect 
(A)

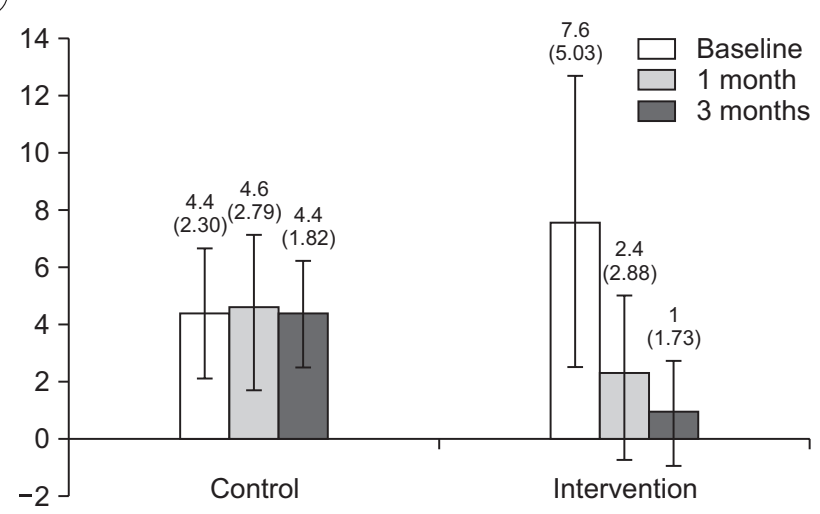

(C)

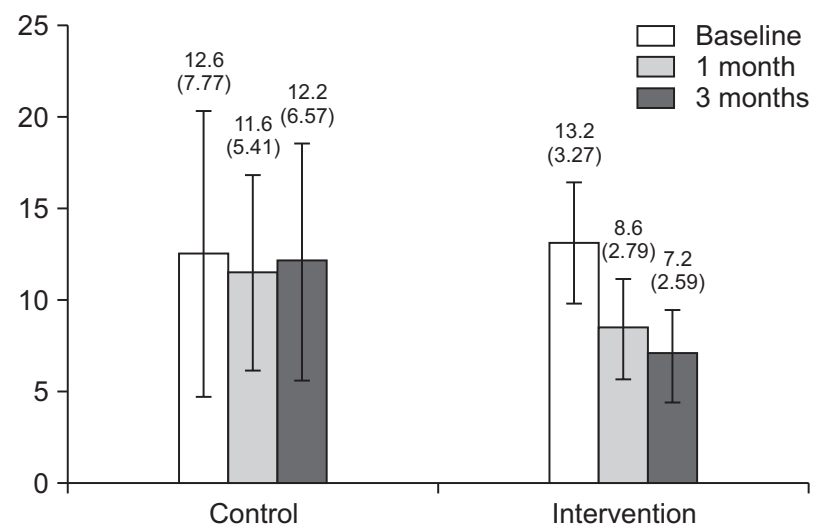

(B)

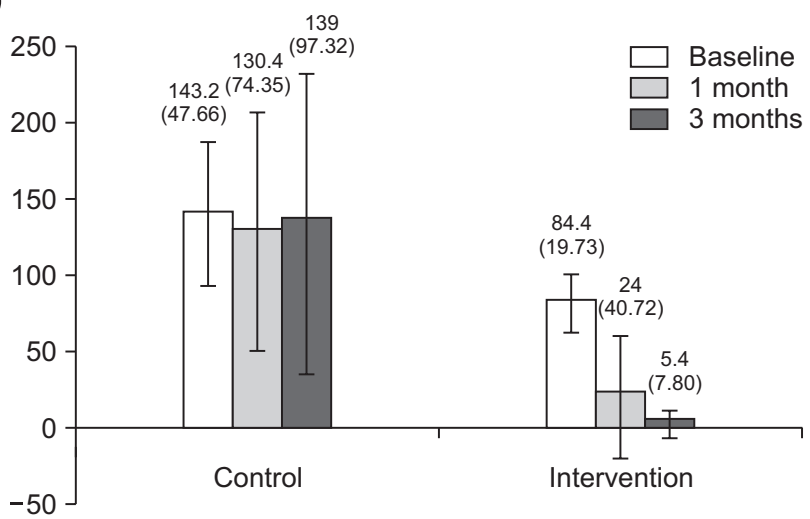

(D)

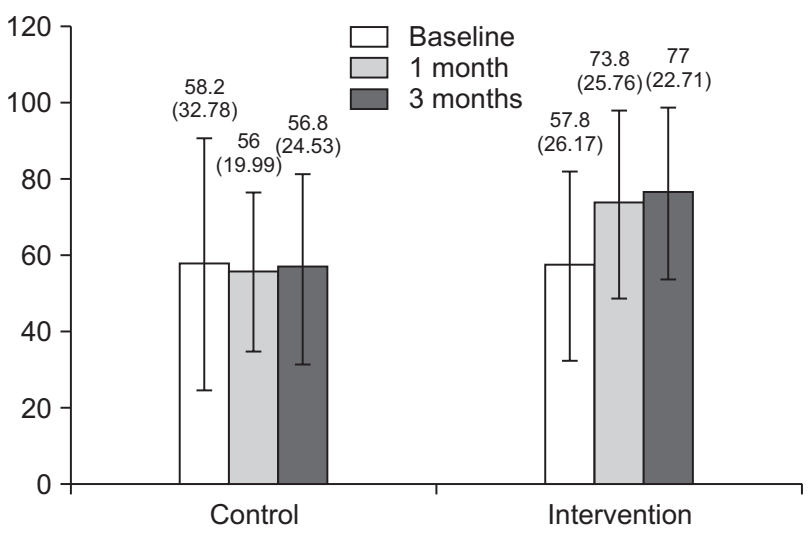

Fig. 2. A comparison of daily frequency and volume of urinary incontinence and ICIQ and IQOL scores at baseline and at 1 and 3 months after intervention. (A) Frequency of urinary incontinence, (B) volume of urinary incontinence, (C) ICIQ, and (D) IQOL. ICIQ, International Consultation on Incontinence Questionnaire; IQOL, urinary incontinence quality of life scale. Values are presented as mean (standard error).

Table 3. Values of UDS parameters at baseline and follow-up and pre/post differences in the intervention and control groups

\begin{tabular}{|c|c|c|c|c|c|c|c|}
\hline \multirow{2}{*}{ Variable } & \multicolumn{3}{|c|}{ Control group } & \multicolumn{3}{|c|}{ Intervention group } & \multirow{2}{*}{$\mathrm{Z}$ (p-value) } \\
\hline & Baseline & $3 \mathrm{mo}$ & Post-Pre & Baseline & $3 \mathrm{mo}$ & Post-Pre & \\
\hline $\begin{array}{l}\text { RDV } \\
(\mathrm{mL})\end{array}$ & $181.60 \pm 81.09$ & $205.80 \pm 42.33$ & $9.75 \pm 13.65$ & $198.60 \pm 84.67$ & $285.00 \pm 70.15$ & $-85.75 \pm 92.59$ & $\begin{array}{l}-2.310 \\
(0.029)^{*}\end{array}$ \\
\hline $\begin{array}{l}\mathrm{MDP} \\
\left(\mathrm{cmH}_{2} \mathrm{O}\right)\end{array}$ & $64.60 \pm 49.97$ & $77.00 \pm 23.82$ & $-5.25 \pm 15.65$ & $80.00 \pm 18.72$ & $63.50 \pm 14.06$ & $19.75 \pm 17.12$ & $\begin{array}{l}-1.440 \\
(0.200)\end{array}$ \\
\hline $\begin{array}{l}\text { Volume at } \\
\text { MDP (mL) }\end{array}$ & $207.20 \pm 163.90$ & $222.80 \pm 48.01$ & $13.25 \pm 16.38$ & $321.80 \pm 163.92$ & $373.30 \pm 63.89$ & $-119.75 \pm 100.31$ & $\begin{array}{l}-2.310 \\
(0.029)^{*}\end{array}$ \\
\hline $\operatorname{MCC}(\mathrm{mL})$ & $331.00 \pm 46.02$ & $351.30 \pm 46.23$ & $-2.50 \pm 8.89$ & $432.40 \pm 118.67$ & $472.80 \pm 39.63$ & $-82.25 \pm 61.44$ & $\begin{array}{l}-1.443 \\
(0.200)\end{array}$ \\
\hline
\end{tabular}

Values are presented as mean \pm standard deviation.

UDS, urodynamic study; RDV, reflex detrusor volume at first contraction; MDP, maximum detrusor pressure during filling; MCC, maximum cystometric capacity.

*Statistically significant at the $\mathrm{p}<0.05$. 
(Fig. 2).

Values of UDS parameters at baseline and follow-up and pre/post differences in both groups are shown in Table 3. Differences in volume at maximal detrusor pressure during filling and reflex detrusor volume at first contraction exhibited significant intergroup differences $(p<0.05)$, whereas maximum cystometric capacity and maximum detrusor pressure during filling did not ( $p>0.05)$.

\section{DISCUSSION}

Contraction of the urinary bladder that causes bladder emptying is controlled by parasympathetic segments that originate from the $2 \mathrm{nd}$, 3rd, and 4th sacral cord segments, and the segments of this nerve innervate the visceral motor neurons in the parasympathetic ganglia around the bladder wall. In patients with spinal cord injury who have lost descending control of the sacral spinal cord, autonomic regulation of bladder continues, and the micturition is simulated reflexively at the level of the sacral cord. However, this reflex is ineffective when descending motor control is damaged and causes urinary dysfunction [16]. It has been demonstrated that removing the sacral reflex arc might liberate the bladder from any possible stimulatory or inhibitory impulses from higher centers [17]; as such, selective sacral rhizotomy has been performed to increase bladder capacity and preserve detrusor reflex and sphincter function in patients with neurogenic bladder.

RF ablation ablates nerves by generating heat via a high-frequency alternating current [18]. In the present study, we used this technique to ablate both the S2 and S3 nerves using bipolar electrodes. In a previous study, monopolar CRF was performed on sacral nerves in spinal cord injury patients with neurogenic bladder [9]. Monopolar RF has been widely used in neurosurgery and for pain management, but bipolar RF has not been used frequently and is less well understood. However, it is known that bipolar RF might be more effective than monopolar RF because whereas monopolar RF generates an RF current between an electrode's exposed tip and a distant ground pad, bipolar RF generates RF current between two closely positioned electrode tips, which allows for higher RF electrical fields to be generated, more rapid tissue heating, and the ability to generate larger lesions than is possible by monopolar RF [19-21].
In the current study, we investigated the effect of bipolar CRF on sacral nerves to treat neurogenic detrusor hyperactivity patients with spinal cord injury. We expected to find that intervention would increase bladder capacity and reduce uninhibited bladder contraction. Improvements in daily mean frequency and volume of urinary incontinence and in ICIQ and IQOL scores were observed in the intervention group compared with the control group with respect to time, which indicates that these variables showed significant changes regardless of a group effect. Additionally, in the group $\times$ time interaction, frequency of urinary incontinence and ICIQ and IQOL scores achieved statistical significance, indicating that these variables showed significant differences between the two groups according to time. In addition, there were significant intergroup differences in pre/post volume differences at maximal detrusor pressures during filling and in reflex detrusor volumes, but pre/post differences in maximal detrusor pressure during filling and maximal cystometric capacity were not significantly different. We believe that these results might be attributable to the small number of subjects we recruited. Nevertheless, bipolar CRF ablation of sacral nerves would appear to be effective in patients with spinal cord injury with hyperactive neurogenic bladder because it improves QOL by attenuating the frequency of urinary incontinence. In fact, all patients had used medications for neurogenic bladder, but medication use after intervention changed only in the intervention group; two patients reduced their use of anticholinergics from two medicines to one; one patient ceased to use any anticholinergics; one reduced anticholinergic use from four to two medicines; and one reduced anticholinergic use from three to two medicines.

Other favorable effects were found in the intervention group. First, three patients reported improvements in defecation. Before intervention, they had typically defecated every four or five days on laxatives and took more than 30 minutes per defecation, whereas after intervention, they defecated every one or two days and took within 30 minutes without laxatives. Although the pathogenetic mechanism that was responsible for this effect is not known, we suggest that RF applied to sacral nerves might have caused denervation of the pudendal nerve and reduce anal sphincter tone. Furthermore, reduced use of anticholinergics would have affected defecation in the intervention group. Second, one patient in the intervention 
group mentioned that neuropathic pain in the inguinal area was improved after intervention. He reported feeling an uncomfortable pain, described as a sharp tingling sensation in the inguinal area before intervention, which was nearly resolved after intervention. Third, another patient in the intervention group reported that symptoms of autonomic dysreflexia were resolved. He usually experienced symptoms described as a pounding headache, flushing, and sweating before intervention, but he found that the symptom of intervention autonomic dysreflexia completely resolved after intervention.

Percutaneous RF is more cost-effective and less invasive than other procedures that are used to treat neurogenic bladder, such as neuromodulation therapy. Neuromodulation, which requires that after successful sacral nerve stimulation, a generator and lead be implanted, is known to be an effective therapy in patients with hypertonic bladder that is responsive to conservative treatment. However, the cost of initial therapy is $\$ 1,800$ to test sacral nerve stimulation, and for ongoing therapy, the cost of a sacral nerve stimulation surgical implant is in the area of $\$ 23,000$ [22]. In contrast, the cost of applying bipolar CRF to sacral nerves is $\$ 300-\$ 400$, and thus, bipolar CRF is most likely the most cost-effective therapy for spinal cord injury patients with neurogenic bladder.

In conclusion, this study shows that applying percutaneous bipolar CRF to sacral nerves offers an effective means for treating neurogenic overactive bladder and that it reduces urinary incontinence and improves quality of life. In addition, RF is relatively safe, cost-effective and repeatable. Furthermore, no serious side effects, such as intervention site infection or serious erectile dysfunction, were encountered after intervention. Although some members of the intervention group experienced discomfort around the intervention site after intervention, they reported that it had lasted only a few days. The major limitation of this study is the small number of subjects enrolled. Furthermore, we evaluated results at only three months after intervention. Accordingly, we suggest that additional larger-scale, longer follow-up studies be conducted.

\section{CONFLICT OF INTEREST}

No potential conflict of interest relevant to this article was reported.

\section{REFERENCES}

1. Ku JH. The management of neurogenic bladder and quality of life in spinal cord injury. BJU Int 2006;98:739-45.

2. Hansen RB, Biering-Sorensen F, Kristensen JK. Urinary incontinence in spinal cord injured individuals 10-45 years after injury. Spinal Cord. 2010;48:27-33.

3. Lieberman JA 3rd. Managing anticholinergic side effects. Prim Care Companion J Clin Psychiatry 2004;6(Suppl 2):20-3.

4. Wyndaele JJ. Complications of intermittent catheterization: their prevention and treatment. Spinal Cord 2002;40:536-41.

5. Houle AM, Vernet O, Jednak R, Pippi Salle JL, Farmer JP. Bladder function before and after selective dorsal rhizotomy in children with cerebral palsy. J Urol 1998;160(3 Pt 2):1088-91.

6. Toczek SK, McCullough DC, Gargour GW, Kachman R, Baker R, Luessenhop AJ. Selective sacral rootlet rhizotomy for hypertonic neurogenic bladder. J Neurosurg 1975;42:567-74.

7. Brindley GS. The first 500 patients with sacral anterior root stimulator implants: general description. Paraplegia 1994;32:795-805.

8. Mulcahy JJ, Young AB. Percutaneous radiofrequency sacral rhizotomy in the treatment of the hyperreflexic bladder. J Urol 1978;120:557-8.

9. Mulcahy JJ, Young AB. Long-term follow-up of percutaneous radiofrequency sacral rhizotomy. Urology 1990;35:76-7.

10. Ferreira RS, Levi d'Ancona CA, Dantas-Filho VP, Rodrigues Netto N Jr, Miyaoka R. Percutaneous radiofrequency sacral rhizotomy in the treatment of neurogenic detrusor overactivity in spinal cord injured patients. Actas Urol Esp 2011;35:325-30.

11. Cho KH, Lee SS. Radiofrequency sacral rhizotomy for the management of intolerable neurogenic bladder in spinal cord injured patients. Ann Rehabil Med 2012;36:213-9.

12. Avery K, Donovan J, Peters TJ, Shaw C, Gotoh M, Abrams P. ICIQ: a brief and robust measure for evaluating the symptoms and impact of urinary incontinence. Neurourol Urodyn 2004;23:322-30.

13. Oh SJ, Park HG, Lim SH, Hong SK, Martin ML, Ting $\mathrm{BL}$, et al. Translation and linguistic validation of Ko- 
rean version of the Incontinence Quality of Life (IQoL) instrument. J Korean Continence Soc 2002;6:1023.

14. Wagner TH, Patrick DL, Bavendam TG, Martin ML, Buesching DP. Quality of life of persons with urinary incontinence: development of a new measure. Urology 1996;47:67-72.

15. van Leijsen SA, Kluivers KB, Mol BW, Broekhuis SR, Milani FL, van der Vaart $\mathrm{CH}$, et al. Protocol for the value of urodynamics prior to stress incontinence surgery (VUSIS) study: a multicenter randomized controlled trial to assess the cost effectiveness of urodynamics in women with symptoms of stress urinary incontinence in whom surgical treatment is considered. BMC Womens Health 2009;9:22.

16. Purves D, Augustine GJ, Fitzpatrick D, Katz LC, Lamantia A, McNamara JO, et al. Neuroscience. 2nd ed. Sunderland: Sinauer Associates Inc.; 2001.

17. Meirowsky AM, Scheibert CD, Hinchey TR. Studies on the sacral reflex arc in paraplegia; response of the bladder to surgical elimination of sacral nerve impulses by rhizotomy. J Neurosurg 1950;7:33-8.

18. Townsend CM, Beauchamp RD, Evers BM, Mattox KL. Sabiston textbook of surgery: the biological basis of modern surgical practice. 18th ed. Philadelphia: Saunders; 2008.

19. Cosman ER, Nashold BS, Ovelman-Levitt J. Theoretical aspects of radiofrequency lesions in the dorsal root entry zone. Neurosurgery 1984;15:945-50.

20. Cosman ER Jr, Cosman ER Sr. Electric and thermal field effects in tissue around radiofrequency electrodes. Pain Med 2005;6:405-24.

21. Cosman ER Jr, Gonzalez CD. Bipolar radiofrequency lesion geometry: implications for palisade treatment of sacroiliac joint pain. Pain Pract 2011;11:3-22.

22. Martinson M, MacDiarmid S, Black E. Cost of neuromodulation therapies for overactive bladder: percutaneous tibial nerve stimulation versus sacral nerve stimulation. J Urol 2013;189:210-6. 